\title{
The Spinnability of Aqueous Polymer Solutions
}

\author{
Kazuhiko HaSHIMOTO and Toyoko IMAE* \\ Department of Chemistry, Faculty of Science, Nagoya University, \\ Chikusa, Nagoya 464, Japan
}

(Received October 19, 1989)

\begin{abstract}
Spinnability has been measured for extract from root of Abelmoschus Manihot and for aqueous solutions of sodium alginate, poly(ethylene oxide) (PEO), and sodium carboxymethylcellulose ( $\mathrm{NaCMC}$ ). The maximum thread length, in other words, drawing length increased linearly with a raising velocity, that is to say, drawing velocity of a rod for extract from root of Abelmoschus Manihot and for aqueous solutions of sodium alginate and PEO. The constancy of the drawing length against drawing velocity was obtained for aqueous solutions of sodium alginate and NaCMC. Such difference in spinnability was discussed in connection with junction formation among polymers in aqueous medium.
\end{abstract}

KEY WORDS Spinnability / Aqueous Polymer Solution / Abelmoschus Manihot / Sodium Alginate / Poly(ethylene oxide) / Sodium Carboxymethylcellulose / Viscoelasticity / Pseudo-Network Theory /

Extract from the root of Abelmoschus Manihot is used to disperse paper fibers on account of its high viscosity, when Japanese paper is manufactured. The main composition of the root of Abelmoschus Manihot is a polysaccharide with molecular weight of $(1-2) \times 10^{5}$, which is composed of rhammnose and galacturonate. ${ }^{1}$ The extract exhibits remarkable viscoelasticity, indicating junction formation among polysaccharide chains.

Aqueous solutions of sodium alginate, $\mathrm{PEO}$, and $\mathrm{NaCMC}$ are also very viscous and can be used to disperse fibers in paper manufacture. Sodium alginate, a major component of brown seaweed, is a linear copolymer of $\alpha$-Lguluronate and $\beta$-D-mannuronate in block and heterogeneous sequences. ${ }^{2-4}$ In an aqueous solution of sodium alginate, the intermolecular linkages mediated by cations are formed..$^{5,6}$ Chains of PEO, a simple and homogeneous polymer, are in the helical form and interact with each other through hydrogen bonding in an aqueous solution. ${ }^{7-10}$ Moderately substituted molecules of $\mathrm{NaCMC}$ in a relatively

\footnotetext{
* To whom all correspondence should be addressed.
}

concentrated solution are jointed by noncovalent linkages, resulting in bundles of chains. ${ }^{11}$

The junction formation in polymer solutions described above should be related to their viscoelastic properties. Spinnability, which is a phenomenon of viscoelasticity, was therefore investigated for aqueous polymer solutions in this paper. Although the spinnability for aqueous solutions of polymeric materials has been reported by some workers, ${ }^{12-14}$ we can distinguish the spinnability of polymer solutions as two types, corresponding to different modes, which has recently been carried out for aqueous surfactant solutions. ${ }^{15} \mathrm{We}$ also discuss two types of spinnability in relation to the conformation of polymers in water.

\section{EXPERIMENTAL}

Extract from the root of Abelmoschus Manihot was kindly provided by Paper Art of Obara, Aichi. Sodium alginate and PEO (molecular weight $\simeq 2 \times 10^{4}$ ) were purchased 
from Scientific Polymer Products, Inc., New York. NaCMC (degree of polymerization $\simeq$ 1050) was purchased from Tokyo Kasei Co., Inc., Tokyo. All samples were used without any purification. Sodium alginate and $\mathrm{NaCMC}$ were dissolved in water at $c a .60^{\circ} \mathrm{C}$.

The apparatus for spinnability measurement was constructed, according as previously reported. ${ }^{12}$ The details are described in elsewhere. ${ }^{15}$ A stainless steel rod of $5 \mathrm{~mm}$ in diameter immersed in a test solution is driven up at a constant rate by motor and pulley. As the rod is pulled up from the solution, a liquid is stretched to form a thread. The thread is broken off when the rod reaches a certain height. This height, drawing length, is measured as the spinnability.

Since the spinnability at a given raising velocity, that is, drawing velocity, $v$, changes linearly with the immersed depth of a rod, the intrinsic drawing length, $L_{0}$, extrapolated to zero immersed depth is presented henceforth.

\section{RESULTS}

The relations between the intrinsic drawing length and drawing velocity of a rod are represented in Figure 1. The intrinsic drawing length at $25^{\circ} \mathrm{C}$ for extract from root of

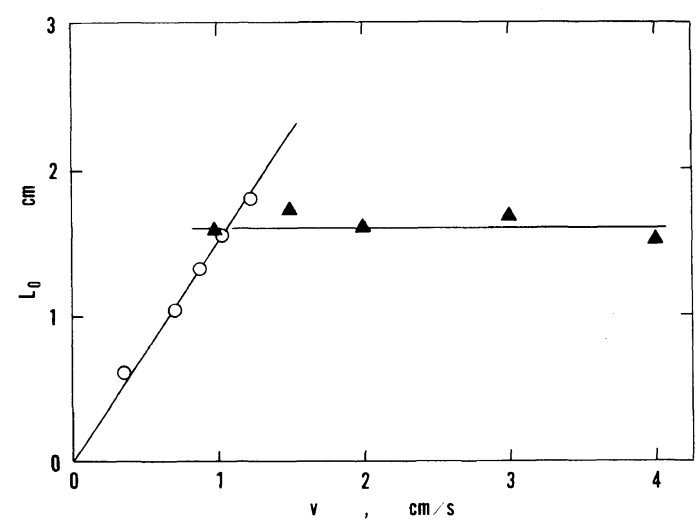

Figure 1. Relations between intrinsic drawing length and drawing velocity for aqueous polymer solutions. $\mathrm{O}$, Abelmoschus Manihot of $0.053 \mathrm{wt} \%$ at $25^{\circ} \mathrm{C} ; \boldsymbol{\Delta}, \mathrm{NaCMC}$ of $2.5 \mathrm{wt} \%$ at $15^{\circ} \mathrm{C}$.
Abelmoschus Manihot of $0.053 \mathrm{wt} \% \quad(\mathrm{~g} / 100 \mathrm{~g}$ solution) increased linearly with the drawing velocity. The slope expressed by $\Delta L_{0} / \Delta v$ characterizes this type of spinnability. The spinnability which exhibits such relation between $L_{0}$ and $v$ is classified as type D. ${ }^{15}$ In the type $\mathrm{D}$ spinnability, the liquid thread becomes thin with drawing till broken. This spinnability is therefore considered to be a ductile-like failure which is characteristic of viscoelastic solutions with low viscosity. ${ }^{16}$

The plots of $\Delta L_{0} / \Delta v$ against concentration, $c$, are given in Figure 2 for the extract from the root of Abelmoschus Manihot at different temperatures. The $\Delta L_{0} / \Delta v$ values increased with increasing concentration. At low concentrations, the $\Delta L_{0} / \Delta v$ values exhibited a slight temperature dependence, whereas they increased with lowering temperature at high concentrations.

Linear plots between the intrinsic drawing length and the drawing velocity were also observed for aqueous solutions of sodium

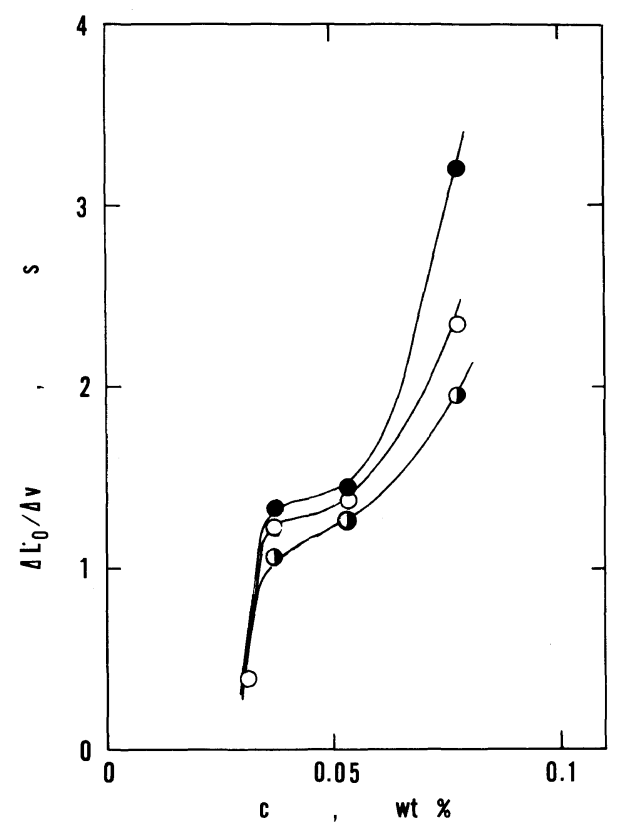

Figure 2. Plots of $\Delta L_{0} / \Delta v v s . c$ for the extract from the root of Abelmoschus Manihot. Temperature $\left({ }^{\circ} \mathrm{C}\right)$ : $\mathbf{0}, 15$; ○, 25; 135 . 


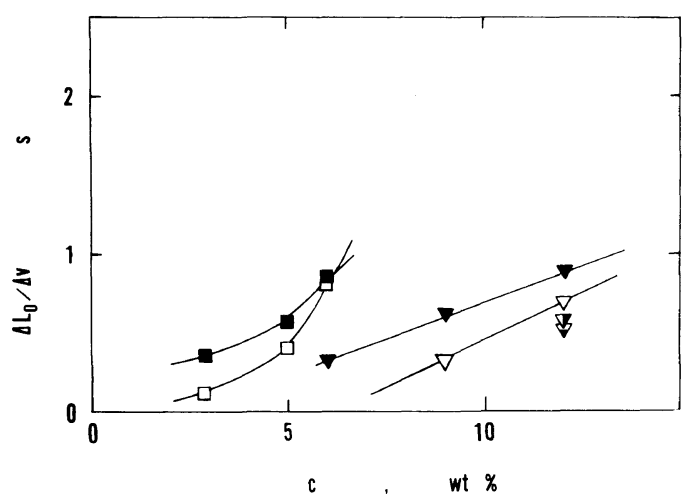

Figure 3. Plots of $\Delta L_{0} / \Delta v$ vs. $c$ for aqueous polymer solutions. Square, sodium alginate; reciprocal triangle, PEO. Temperature $\left({ }^{\circ} \mathrm{C}\right): \mathbf{D}, \boldsymbol{\nabla}, 15 ; \square, \nabla, 25 ; \nabla, 35 ; \nabla, 45$.

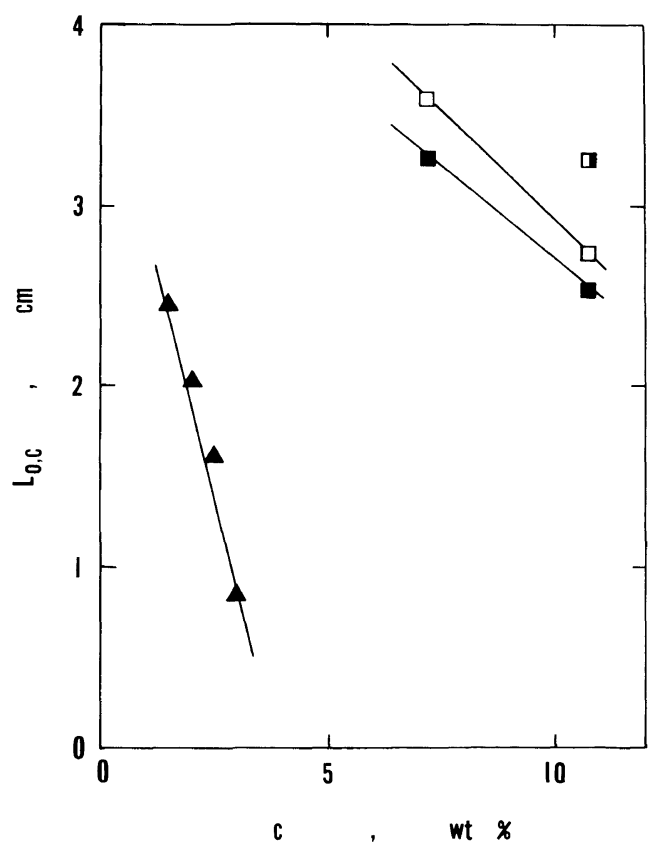

Figure 4. Plots of $L_{0, \mathrm{c}}$ vs. $c$ for aqueous polymer solutions. Square, sodium alginate; triangle, NaCMC. Temperature $\left({ }^{\circ} \mathrm{C}\right): \mathbf{\square}, \mathbf{\Delta}, 15 ; \square, 25 ; \square, 35$.

alginate at low concentrations and of PEO. For these solutions, the $\Delta L_{0} / \Delta v$ values increased with their concentrations and with lowering temperature, as seen in Figure 3, as well as the extract from root of Abelmoschus Manihot.

For aqueous solutions of sodium alginate at high concentrations and of $\mathrm{NaCMC}$, however, the intrinsic drawing length was approximately independent of drawing velocity, as illustrated in Figure 1 for aqueous solution of $\mathrm{NaCMC}$ of $2.5 \mathrm{wt} \%$ at $15^{\circ} \mathrm{C}$. Then the intrinsic drawing length measured at fast drawing, $L_{0, \mathrm{c}}$, can be defined. This type of spinnability is called type C. ${ }^{15}$ The liquid thread in type $\mathrm{C}$ spinnability is almost constant in its thickness during the drawing and snaps at a position near the rod. The type $C$ spinnability which resembles a rubber elasticity or a gel elasticity can be regarded as cohesive-like fracture. ${ }^{16}$ The plots of $L_{0, \mathrm{c}}$ vs. concentration are given in Figure 4. For aqueous solutions of sodium alginate and $\mathrm{NaCMC}$, the $L_{0, \mathrm{c}}$ values decreased with increasing concentration and with cooling.

\section{DISCUSSION}

Extensive measurement for the spinnability of aqueous polymer solutions was performed by Erbring. ${ }^{12} \mathrm{He}$ found that the spinnability did not depend simply on the viscosity of liquid and that the surface tension did not affect the spinnability, whereas non-Newtonian viscosity or viscoelasticity was closely related to the spinnability. Nakagawa ${ }^{14}$ examined type D spinnability of rubber in xylene solution and suggested that the spinnability was an elastic deformation superposed on a viscous flow. The thread was broken when deformation rate coincided with the inverse of the mechanical relaxation time of liquid in a Maxwell body.

The $\Delta L_{0} / \Delta v$ value in type $\mathrm{D}$ spinnability, which is the reciprocal deformation rate of thread when the thread is broken, corresponds to the relaxation time. It is possible to interpret from the stress relaxation experiment ${ }^{17}$ that the relaxation time of aqueous PEO solution increased with its concentration. This is consistent with the finding that the $\Delta L_{0} / \Delta v$ values for aqueous PEO solution increase with the concentration.

The above description can be considered on the basis of pseudo-network theory which has 
been proposed for polymer solutions. ${ }^{18}$ According to the theory, the elongational viscosity increases steeply with increasing elongational velocity. If the tension of thread is uniform along its length, minute shortening of the diameter of the thread by fluctuation strengthens largely the stress so that the local elongational velocity is suppressed. Thus further decrease in diameter is restrained, and the thread in type D spinnability is stabilized.

The liquid thread in type $\mathrm{C}$ spinnability is broken when the adhesion force of thread to a rod is overcome by the tension of the thread that is determined by the modulus and strain. Then the $L_{0, \mathrm{c}}$ value in type $\mathrm{C}$ spinnability may reflect the modulus of the solution. It is, therefore, expected that the $L_{0, \mathrm{c}}$ values decrease as the modulus increases. The $L_{0, \mathrm{c}}$ values for aqueous solutions of sodium alginate at high concentrations and of $\mathrm{NaCMC}$ decrease with concentration. This result represents that the modulus of these polymer solutions increases with concentration. The rheology of NaCMC has been studied extensively, ${ }^{19-21}$ and Lindbery et al. $^{20}$ and Thurston and Martin ${ }^{21}$ found that the dynamic modulus increased with increasing concentration. Their reports support the explanation described above.

Many investigations for the viscoelasticity of aqueous polymer solutions suggest that intermolecular interactions participate in viscoelastic properties of polymer solutions. The $\mathrm{Na}^{+}$-induced intermolecular association was proposed for sodium alginate in water. ${ }^{6}$ Less charged parts of $\mathrm{NaCMC}$ chains aggregate and make bundles, while considerably charged free parts of chains spread out; some NaCMC chains extend through bundles. ${ }^{11}$ Molecules of $\mathrm{PEO}$ and $\mathrm{NaCMC}$ are strongly hydrated in aqueous solutions. ${ }^{7,8,22}$ Hydration may be involved in the intermolecular interaction through hydrogen bonding between polymer molecule and water. These intermolecular interactions can make higher-order structures and participate not only in viscous property but also in elastic response of polymer solutions. For example, some polyelectrolytes were observed to form a complex, aggregate, and network. ${ }^{23-25}$

With increasing concentration, polyanions tend to take a shrunk configuration and to promote the intermolecular association between them. ${ }^{19}$ Therefore, viscoelastic properties of such polymer solutions may change considerably with concentration, so that the relaxation time lengthens and the modulus rises.

The difference between types C and D spinnability may depend on life time, amount and strength of junctions among polymers, on the concentration of free and interacting polymer chains and on the dimension of linked aggregate. If linkages are few or weak, the type D spinnability should appear, because the solution is easy to deform and flow. The stress necessary for type $\mathrm{C}$ spinnability was then dissipated. The rheological properties of the solutions corresponded to these of ordinary polymer solutions. On the other hand, when most of the polymers interacted strongly and very large linked aggregates were formed, the type $\mathrm{C}$ spinnability should be superior, since a higher-order structure brought forth elastic response and flowing of the solution was difficult. The stress resulting in type $\mathrm{C}$ spinnability is thus maintained.

\section{CONCLUSIONS}

It is concluded that the spinnability of aqueous polymer solutions can be classified into two types, based on the dependence on drawing velocity. The $\Delta L_{0} / \Delta v$ value in type $\mathrm{D}$ spinnability is connected with the relaxation time of a solution which increases with concentration. On the other hand, the $L_{0, \mathrm{c}}$ value in type $\mathrm{C}$ spinnability reflects the elastic modulus of a solution. It decreases with an increase in modulus which increases with concentration. The spinnability may be related to junction formation among polymers. The 
type D spinnability appears in a solution with few or weak junction linkages among polymers, while the type $\mathrm{C}$ spinnability is superior in strongly interacting polymer solutions.

\section{REFERENCES}

1. S. Ikawa, R. Goto, and I. Morimoto, Nihon Kagaku Zasshi (Japan), 81, 783 (1960).

2. A. Haig, B. Larsen, and O. Smidsrod, Acta Chem. Scand., 20, 183 (1966).

3. A. Haig, B. Larsen, and O. Smidsrod, Acta Chem. Scand., 21, 691 (1967).

4. A. Haig, B. Larsen, and O. Smidsrod, Carbohydr. Res., 23, 563 (1974).

5. E. R. Morris and D. A. Rees, J. Mol. Biol., 138, 363 (1980).

6. R. Seale, E. R. Morris, and D. A. Rees, Carbohydr. Res., 110, 101 (1982).

7. K-J. Liu, Macromolecules, 1, 213 (1968).

8. K-J. Liu and J. L. Parsons, Macromolecules, 1, 529 (1969).

9. A. Sarkar and K. Ghosh, Kolloid-Z. Z. Polym., 236, 140 (1970).

10. N. B. Graham, M. Zulfiqar, N. E. Nwachuku, and A. Rashid, Polymer, 30, 528 (1989).
11. D. A. Rees, Adv. Carbohydr. Chem. Biochem., 24, 267 (1969).

12. H. Erbring, Koll. Beih., 44, 171 (1936).

13. H. Thiele and H. Lamp, Kolloid Z., 129, 25 (1952).

14. T. Nakagawa, Bull. Chem. Soc. Jpn., 25, 88, 93 (1952).

15. T. Imae, K. Hashimoto, and S. Ikeda, Colloid Polym. Sci., in press.

16. Y. Ide and J. L. White, J. Appl. Polym. Sci., 20, 2531 (1976).

17. E. T. Lance-Gomez and T. C. Ward, J. Appl. Polym. Sci., 31, 333 (1986).

18. A. S. Lodge, "Elastic Liquids," Academic Press, London, 1964.

19. S. Kuroiwa, M. Ogawa, and M. Nakamura, Bull. Chem. Soc. Jpn., 40, 2195 (1967).

20. G. B. Thurston and A. Martin, J. Pharm. Sci., 67, 1499 (1978).

21. J. J. Lindberg, H. Sirviö, and J. Martinmaa, Cellulose Chem. Technol., 21, 379 (1987).

22. C. A. Kumsah, G. Pass, and G. O. Phillips, J. Solution Chem., 5, 799 (1976).

23. E. Tsuchida, K. Abe, and M. Honma, Macromolecules, 9, 112 (1976).

24. H. Ohno, H. Matsuda, and E. Tsuchida, Makromol. Chem., 182, 2267 (1981).

25. H. Ohno, H. Takinishi, and E. Tsuchida, Makromol. Chem., Rapid Commun., 2, 511 (1981). 